Journal of Computer Science and Cybernetics, V.26, N.4 (2010), 361-373

\title{
WALKING PLANNING FOR BIPED ROBOT HUBOT-1
}

\author{
THANH NHUT NGUYEN, TAN LAM CHUNG \\ Robotics and Mechatronics Division, National Laboratory of Digital Control \\ and System Engineering HCMC University of Technology, Vietnam
}

\begin{abstract}
In this paper, an offline trajectory generation is applied to biped robot HUBOT-1. The parameters with constraints for foot motions are formulated. The hip and ankle trajectories are planned. Different foot motions are produced, and the motion which satisfies stable constraints is chosen for deriving the joint actuators. The simulation is given to show the possibility of the planning method.

Tóm tắt. Trong bài báo này, phương pháp hoạch định quỹ đạo offline được ứng dụng vào robot hai chân HUBOT-1. Các thông số của robot với các điều kiện ràng buộc được sứ dụng để hoạch định quỹ đạo di chuyển của bàn chân và hông. Từ quỹ đạo này các dạng di chuyển bước đi khác nhau của robot được tạo ra và các chuyển động này thõa mãn điều kiện cân bằng ZMP được chọn. Các kết quả này được sử dụng để tính toán động cơ sử dụng. Mô phỏng cho thấy khả năng ứng dụng của phương pháp này trên robot thật.
\end{abstract}

\section{INTRODUCTION}

Biped robots have been widely studied by science departments at universities and the industry for years. The objective of research works is to improve stable mobility and intelligence of biped robot and make them useful in life. This type of robots can perform heavy or dangerous work previously imposed on humans. Up to now, four advanced typical biped robots have been presented: ASIMO, HRP-4, Wabian-2R [1] and Johnnie [2]. For the mobility, many related issues such as stability criterion [3,4] were considered. Some researchers [58] proposed the methods for deriving the hip trajectory to execute a desired ZMP. The drawback of these methods is that not all desired ZMP trajectories can be attained and the hip acceleration may need to be very large.

The ZMP criterion is the most widely accepted and used stability measure for the biped robot locomotion. To ensure the dynamic stability of this robot, Takanishi et al. have proposed methods of walking pattern synthesis based on zero moment point, which stays within the convex hull of all contact points between the feet and the ground [9]. Basically, these investigations first design a desired ZMP trajectory, then derive the hip motion or torso motion required to achieve that ZMP trajectory. Since the change of the ZMP due to body motion is limited, not all desired ZMP trajectories can be achieved. In addition, the hip acceleration may need to be very large to achieve a desired ZMP trajectory. In this case, since the torso is 
relatively massive, energy consumption increases, and control for task execution of the upper limbs becomes difficult. Therefore, it is desirable to obtain hip motion without first designing the desired ZMP trajectory [10]. Shih presented a method for producing foot trajectories by 3rd order spline interpolation [11]. When the stability constraint and the ground conditions are satisfied, it is additionally desirable to select a walking pattern that requires small torque and velocity of the joint actuators. Huang et al. [10] proposed a method in which the constraints of a foot and hip trajectory are formulated and this trajectory is generated by a 3rd order spline interpolation with high stability by means of an iterative ZMP calculation. Erbatur et al. [12] proposed a method to obtain naturalness in the walk by using Fourier series approximation techniques for the solution of LIPM dynamics equations.
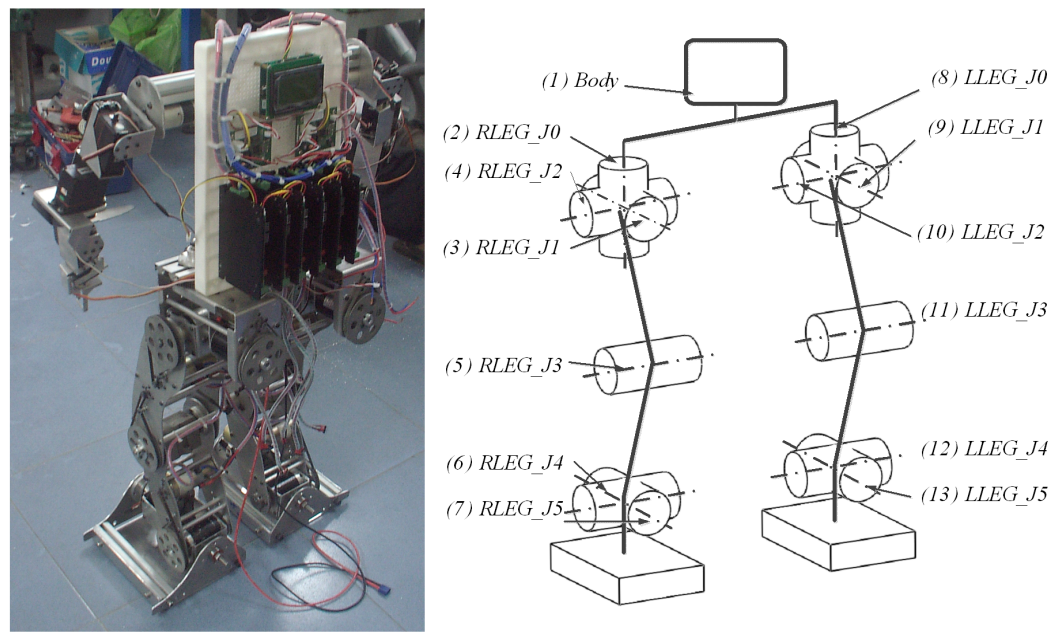

Fig. 1. a) Picture of HUBOT b) Model of the biped robot

This paper uses the method proposed in [10] to robot HUBOT-1 for planning walking patterns in sagittal plane and propose a method of y-trajectory generation based on CoM trajectory from [12], which includes the ground conditions, dynamic stability constraint, and relationship between walking patterns and actuator specifications.

This paper is organized as follows. The gait cycle is presented in Section 2. The gait planing and balanced control used in the simulations is given in Section 3. Section 4 is devoted to simulation results with the generated reference trajectories.

\section{GAIT CYCLE}

Our biped robot (Fig. 1) consists of an aluminum skeleton with six DOF on one leg: three DOF in the hip joint, one in the knee, and two in the ankle. The robot should have the same characteristics in the walking pattern as a human. Therefore the human walking pattern will be described by gait cycle, the movement of the body by putting each foot in front in turns, while both feet never leave the ground at the same time. 
The human gait consists of a repeatable cycle which is described by two phases: a doublesupport phase (DSP) and a single-support phase (SSP). The cycle start the foot becomes the rearmost foot leaves the ground and becomes the front foot again. During the double-support phase, both feet are in contact with the ground. During the single-support phase, while one foot is stationary on the ground, the other foot swings from the rear to the front. (Fig. 2)

If both foot trajectories and the hip trajectory are known, all joint trajectories of the biped robot will be determined by kinematic constraints uniquely. When the robot moves straightforward, the lateral positions of both feet are constant. The lateral hip motion is consider with y-dimensional for natural walking.

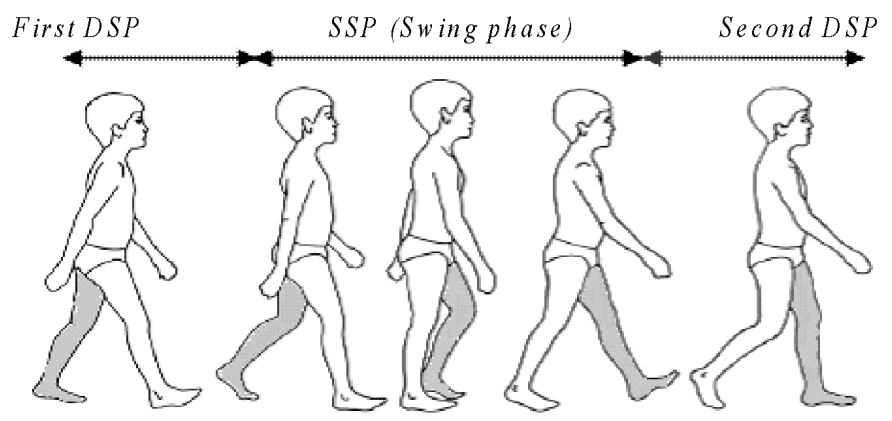

Fig. 2. The human gait cycle

Biped robots are often modeled as inverted pendulums to reduce the complexity of the mechanical model. A 3D-Linear Inverted Pendulum method is used when the mass of the robot is assumed to be concentrated in one point. This assumption is justified when the main body, the head and the arms amount is more than $70 \%$ of the total robot mass. HUBOT-1 is not satisfied the above assumption, this is the reason why this method can not be applied. Instead, the GCoM method is often applied to generate trajectories. The method works by moving the CoM from one foot to the other one and vice versa. It is a relative simple way for stable walk, but it makes only possible to walk static and not dynamic gait as a human. However, this method is suitable for the first test of our biped robots, velocities are very slow, which makes it easier to follow the individual joints to verify whether they follow the trajectory.

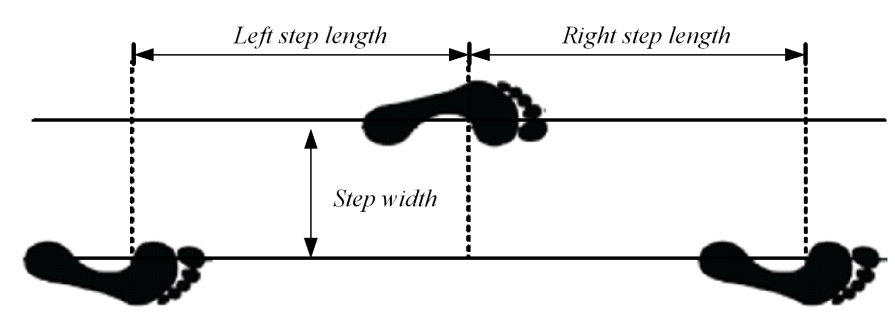

Fig.3. Geometrical measures of gait 


\section{GAIT PLANNING}

This strategy of gait in sagittal plane is totally based on [10] for the comprehensive analysis in general case of ground condition, obstacle-filled environment; but in lateral plane, hip motion in y-dimensional based on CoM trajectory from [12], the gait planning is summarized as the followings:

For a sagittal plane, each foot trajectory can be denoted by a vector as follows:

$$
X_{a}=\left[x_{a}(t), z_{a}(t), \theta_{a}(t)\right]^{T}
$$

where

$$
\begin{aligned}
& \left(x_{a}(t), z_{a}(t)\right): \text { the coordinate of the ankle position } \\
& \theta_{a}(t): \text { the angle of the foot. }
\end{aligned}
$$

The hip trajectory in three dimensional can be denoted by a vector:

$$
X_{h}=\left[x_{h}(t), y_{h}(t), z_{h}(t), \theta_{h}(t)\right]^{T}
$$

$\left(x_{h}(t), y_{h}(t), z_{h}(t)\right)$ : the coordinate of the hip position

$\theta_{h}(t)$ : the angle of the hip.

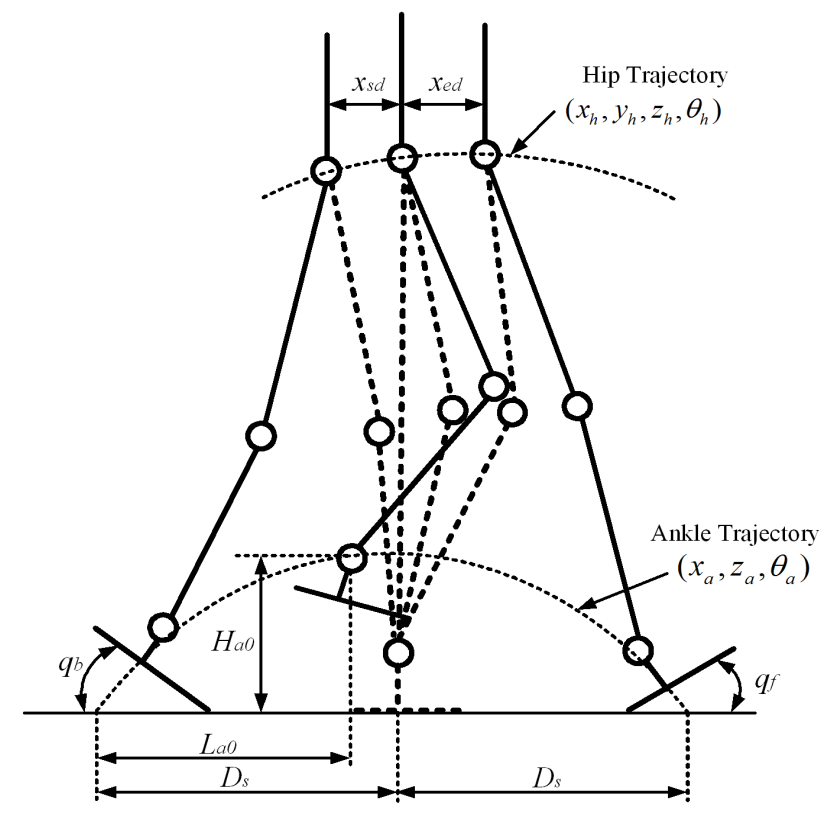

Fig. 4. Walking parameters [10]

In order to enable the robot to adapt to various ground conditions, we must first specify both foot trajectories, and then determine the hip trajectory.

\subsection{Foot Trajectory}

Assuming that the period necessary for one walking step is $T_{C}$, the time of the step is from $k T_{C}$ to $(k+1) T_{C}, k=1,2, . ., k, k$ is the number of steps. To simplify our analysis, we define 
the walking step to begin with the heel of the right foot leaving the ground at $t=k T_{C}$, and to end with the heel of the right foot making first contact with the ground at $t=(k+1) T_{C}$. In this paper, the feet are always level with the ground, that means, the foot angle is always zero for the simplicity, though it is not the case for natural human locomotion and aesthetics and high-speed walking.

Assuming that the entire sole surface of the right foot is in contact with the ground at $t=k T_{C}$ and $t=(k+1) T_{C}$, we get the following constraints:

$$
\theta_{a}(t)= \begin{cases}q_{g s}(k), & t=k T_{c} \\ q_{b}, & t=k T_{c}+T_{d} \\ -q_{f}, & t=(k+1) T_{c} \\ -q_{g e}(k), & t=(k+1) T_{c}+T_{d}\end{cases}
$$

where

$T_{d}:$ the interval of the double-support phase

$q_{g s}(k) q_{g e}(k)$ : the angles of the ground surface under the support foot.

$q_{b}$ : the angle of foot as it leaves the ground

$q_{f}$ : the angle of foot as it lands on the ground

Letting $\left(L_{a o}, H_{a o}\right)$ be the position of the highest point of the swing foot (Fig. 4), from (1) and the kinematic constraints, the following constraints must be satisfied:

$$
x_{a}(t)= \begin{cases}k D_{s}, & t=k T_{c} \\ k D_{s}+l_{a n} \sin q_{b}+l_{a f}\left(1-\cos q_{b}\right), & t=k T_{c}+T_{d} \\ k D_{s}+L_{a o}, & t=k T_{c}+T_{m} \\ (k+2) D_{s}-l_{a n} \sin q_{f}-l_{a b}\left(1-\cos q_{f}\right), & t=(k+1) T_{c} \\ (k+2) D_{s}, & t=(k+1) T_{c}+T_{d}\end{cases}
$$

where

$k$ : number of step

$T_{c}:$ step period

$T_{d}$ : the interval of the double-support phase

$k T_{c}+T_{m}:$ the time of right foot at its highest point

$D_{s}$ : the length of one step

$l_{a n}$ : the height of the foot

$l_{a f}:$ the length from the ankle joint to the toe

$l_{a b}:$ the length from the ankle joint to the heel

$$
z_{a}(t)= \begin{cases}h_{g s}(k)+l_{a n}, & t=k T_{c} \\ h_{g s}(k)+l_{a f} \sin q_{b}+l_{a n} \cos q_{b}, & t=k T_{c}+T_{d} \\ H_{a o}, & t=k T_{c}+T_{m} \\ h_{g e}(k)+l_{a b} \sin q_{f}+l_{a n} \cos q_{f}, & t=(k+1) T_{c} \\ h_{g e}(k)+l_{a n}, & t=(k+1) T_{c}+T_{d}\end{cases}
$$

Since the entire sole surface of the right foot is in contact with the ground at and, the following derivative constraints must be satisfied: 


$$
\begin{aligned}
& \left\{\begin{array}{l}
\dot{\theta}_{a}\left(k T_{c}\right)=0 \\
\dot{\theta}_{a}\left((k+1) T_{c}+T_{d}\right)=0
\end{array}\right. \\
& \left\{\begin{array}{l}
\dot{x}_{a}\left(k T_{c}\right)=0 \\
\dot{x}_{a}\left((k+1) T_{c}+T_{d}\right)=0
\end{array}\right. \\
& \left\{\begin{array}{l}
\dot{x_{a}}\left(k T_{c}\right)=0 \\
\dot{x_{a}}\left((k+1) T_{c}+T_{d}\right)=0
\end{array}\right.
\end{aligned}
$$

To generate a smooth trajectory, it is necessary that the first derivative (velocity) terms $\dot{x}_{a}(t), \dot{z}_{a}(t)$, and $\dot{\theta}_{a}(t)$ be differential, the second derivative (acceleration) terms $\ddot{x}_{a}(t), \ddot{z}_{a}(t)$ and $\ddot{\theta}_{a}(t)$ be continuous at all, including all breakpoints, $t=k T_{C}, k T_{C}+T_{d}, k T_{C}+T_{m},(k+$ 1) $T_{C},(k+1) T_{C}+T_{d}$.

The constraints (3)(8) make the computation difficult to archive using polynomial interpolation, so the foot trajectory can be derived by third-order spline interpolation. In this case $x_{a}(t), z_{a}(t)$ and $\theta_{a}(t)$ are characterized by third-order polynomial expressions, and the second derivatives $\ddot{x}_{a}(t), \ddot{z}_{a}(t)$, and $\ddot{\theta}_{a}(t)$ are always continuous. By varying the values of constraint parameters $q_{g s}(k), q_{g e}(k), h_{g s}(k), h_{g e}(k), q_{b}, q_{f}, H_{a o}$ and $L_{a o}$, we can produce different foot trajectories.

\subsection{Hip Trajectory}

The hip motion of the steady phase is determined in 3D space. The hip motion of the starting phase and the ending phase can be obtained similarly.

First, there is no waist joint in this case, and $\theta_{h}(t)$ is constant:

$$
\theta_{h}(t)=0.5 \pi \mathrm{rad}
$$

Second, hip motion $z_{h}(t)$ of hardly affects the position of the ZMP. Assuming that the hip is at its highest position $H_{h \max }$ at the middle of the single-support phase and at its lowest position $H_{h \min }$ at the middle of the double-support phase:

$$
z_{h}(t)= \begin{cases}H_{h \min }, & t=k T_{c}+0.5 T_{d} \\ H_{h \max }, & t=k T_{c}+0.5\left(T_{c}-T_{d}\right) \\ H_{h \min }, & t=(k+1) T_{c}+0.5 T_{d}\end{cases}
$$

the change of $x_{h}(t)$ and $y_{h}(t)$ is the main factor that affects the stability of a biped robot walking. In this paper, $y_{h}(t)$ is determined from natural CoM references presented in [12]

$$
y_{h}(t)=\frac{a_{0}}{2}+\sum_{k=1}^{\infty}\left[a_{k} \cos \left(\frac{2 \pi k t}{2 T_{c}}\right)+b_{k} \sin \left(\frac{2 \pi k t}{2 T_{c}}\right)\right],
$$

the Fourier coefficients of $y_{h}(t)$ can be obtained as 


$$
\begin{aligned}
& a_{0}=0, \\
& a_{k}=0, \\
& b_{k}=\frac{2 A T_{c}^{2} \omega_{n}^{2}(1-\cos k \pi)}{k \pi\left(T_{c}^{2} \omega_{n}^{2}+k^{2} \pi^{2}\right)}, k=1,2,3, \ldots, \\
& \omega_{n}=\sqrt{g / z_{c}} .
\end{aligned}
$$

In this equation, $A$ is the distance between the foot centers in the y-directional, $g$ is the gravity constant $(9.8 \mathrm{~m} / \mathrm{s} 2)$ and $z_{c}$ is the height of the plane on which the motion of the point mass is constrained, with this robot model $z_{c}$ is chosen equal $z_{h}$.

Fourth, $x_{h}(t)$ must satisfy

$$
x_{h}(t)= \begin{cases}k D_{s}+x_{e d}, & t=k T_{c}, \\ (k+1) D_{s}-x_{s d}, & t=k T_{c}+T_{d}, \\ (k+1) D_{s}+x_{e d}, & t=(k+1) T_{c} .\end{cases}
$$

To obtain a smooth periodic $x_{h}(t)$ of the steady phase using third-order spline interpolation, the following derivative constraints must satisfy:

$$
\left\{\begin{array}{l}
\dot{x}_{h}\left(k T_{c}\right)=\dot{x}_{h}\left(k T_{c}+T_{c}\right) \\
\ddot{x}_{h}\left(k T_{c}\right)=\ddot{x}_{h}\left(k T_{c}+T_{c}\right)
\end{array}\right.
$$

$x_{s d}$ and $x_{e d}$ are hip parameters and changed for the body stability. The values $x_{s d}$ and $x_{e d}$ are specified within a fixed range to get a series of smooth $x_{h}(t)$ as follows:

$$
\left\{\begin{array}{l}
0.0<x_{s d}<0.5 D_{s} \\
0.0<x_{e d}<0.5 D_{s}
\end{array}\right.
$$

A smooth trajectory $x_{h}(t)$ with the largest stability margin can be formulated as follows:

$$
\max _{x_{e d} \in\left(0,0.5 D_{s}\right) ; x_{s d} \in\left(0,0.5 D_{s}\right)} d_{z m p}\left(x_{s d}, x_{e d}\right)
$$

where $d_{z m p}\left(x_{s d}, x_{e d}\right)$ denotes the stability margin.

\subsection{Balanced control}

Vukobratovics ZMP criterion [13] is used for balance control in this simulation. If the ZMP is within the convex hull of all contact points (the stable region), the biped robot is able to walk.

The ZMP can be calculated by using Newtons law from Robot Motion as:

$$
\begin{aligned}
x_{Z M P} & =\frac{\sum_{i=1}^{n} m_{i}\left(\ddot{z}_{i}+g_{z}\right) x_{i}-\sum_{i=1}^{n} m_{i} \ddot{x}_{i} z_{i}-\sum_{i=1}^{n} I_{i, y} \dot{\omega}_{i, y}}{\sum_{i=1}^{n} m_{i}\left(\ddot{z}_{i}+g_{z}\right)} \\
y_{Z M P} & =\frac{\sum_{i=1}^{n} m_{i}\left(\ddot{z}_{i}+g_{z}\right) y_{i}-\sum_{i=1}^{n} m_{i} \ddot{y}_{i} z_{i}+\sum_{i=1}^{n} I_{i, x} \dot{\omega}_{i, x}}{\sum_{i=1}^{n} m_{i}\left(\ddot{z}_{i}+g_{z}\right)}
\end{aligned}
$$

where $n$ is number of links, $m_{i}$ : mass of link i, $I_{i x}, I_{i y}$ is the inertia components of link $\mathrm{i}, \omega_{i x}, \omega_{i y}$ : the absolute angular velocity components around $\mathrm{x}$-axis and $\mathrm{y}$-axis at center of 
gravity of link i, $g_{z}$ is the gravitational acceleration, $\left(x_{i}, y_{i}, z_{i}\right)$ is the coordinate of the mass center of link i on origin coordinate system.

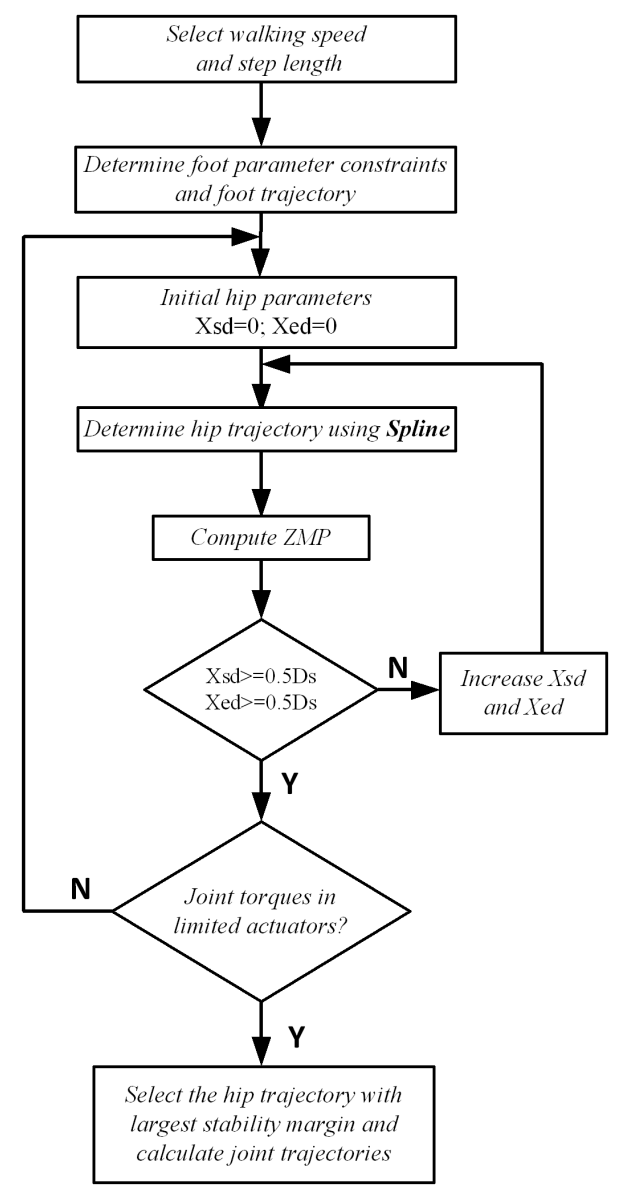

Fig. 5. Algorithm for planning walking patterns [10]

\section{SIMULATION RESULT}

The control strategy in this research is offline motion planning, the walking trajectories are calculated beforehand using an external computer and then implemented on the robot as functions describing the trajectories. The motor control algorithm is a simple one based on independent joint PID position controllers.

The parameters of the biped robot are set according to Table 1. The walking speed is $0.2 \mathrm{~m} / \mathrm{s}$ with the step length of $0.2 \mathrm{~m} / \mathrm{step}$ and the step period of $1 \mathrm{~s} / \mathrm{step}$. To reduce the complexity, the motion planning is at first designed to make the robot walk flat footed, the heel strike and toe off phases can be implemented when the robot is capable of performing this simplified gait. Therefore, level-ground walking condition are as follows:

$q_{g s}(k)=0 \mathrm{rad}, q_{g e}(k)=0 \mathrm{rad}, h_{g s}(k)=0 \mathrm{rad}, h_{g e}(k)=0 \mathrm{rad}$. The position of the highest point of the swing foot $\left(L_{a o}, H_{a o}\right)=(0.1,0.1) m$. Distance between the foot centers in the 
Table 1. Robot parameters (Link of robot in Fig.1b)

\begin{tabular}{|c|c|c|c|}
\hline \multicolumn{2}{|c|}{ Weight of Link (kg) } & \multicolumn{2}{|c|}{ Inertia tensor of Link (kg.cm2) } \\
\hline$m_{1}$ & 3.73 & $I_{1}$ & {$\left[\begin{array}{llllllll}70 & 0.8 & -2.4 ; 0.8 & 51-14.2 ;-2.4 & -14.2 & 30]\end{array}\right.$} \\
\hline$m_{2}=m_{8}$ & 1.73 & $I_{2}=I_{8}$ & {$\left[\begin{array}{llllllll}70 & 0.8 & -2.4 ; 8 & 51 & -4.2 ;-2.4 & -14.2 & 30]\end{array}\right.$} \\
\hline$m_{2}=m_{9}$ & 0.61 & $I_{3}=I_{9}$ & {$\left[\begin{array}{llllllllll}27 & 0 & 0.1 ; & 0 & 4.8 & 0 ; & 0.1 & 0 & 4\end{array}\right]$} \\
\hline$m_{2}=m_{10}$ & 1.79 & $I_{4}=I_{10}$ & [89.6 -4.9 -21; -4.9 53.7 $33.2 ;-2.133 .290 .5$ \\
\hline$m_{5}=m_{11}$ & 1.62 & $I_{5}=I_{11}$ & 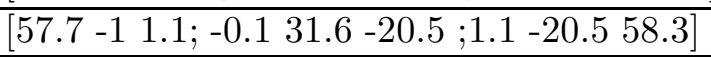 \\
\hline$m_{6}=m_{12}$ & 1.62 & $I_{6}=I_{12}$ & 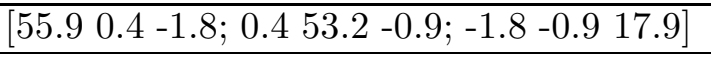 \\
\hline$m_{7}=m_{13}$ & 3.88 & $I_{7}=I_{13}$ & {$\left[\begin{array}{llllllllll}12 & -19.3 & 1 ; & -19.3 & 174.7 & 14.7 ; 1 & 14.7 & 89.3]\end{array}\right.$} \\
\hline
\end{tabular}

y-directional $A=0.25 \mathrm{~m}$. In the simulation, the interval of the double-support phase in human locomotion is about $20 \%$ as the initial value, and this value is varied until the body reaches the walking speed as desired. Highest position $H_{h \max }$ equal lowest position $H_{h \max }=H_{h \min }=$ $0.47 \mathrm{~m}$. Other parameter: $l_{a n}=0.074 m, l_{a b}=0.044, l_{a f}=0.158$, length of thigh: $0.253 \mathrm{~m}$, length of shank: $0.195 \mathrm{~m}$, length of body: $0.5 \mathrm{~m}$.

The hip trajectory is shown in Fig. 6. The ankle trajectory of the left and the right in $\mathrm{X}$ coordinate and $\mathrm{Z}$ coordinate are shown in Fig. $7-8$, respectively. The hip velocity is shown in Fig. 9. It can be seen that the hip and ankle trajectories are smooth enough. The ZMP trajectory that satisfies the largest margin is shown in Fig. 10. The full body motions of the robot in Fig. 9 showed the effectiveness of the simulation as desired. It can be seen that both feet leave and land on the ground with the desired angles of flat-footed walking.

Based on the chosen walking gait, it is also possible to select a walking pattern requiring small specifications of the joint actuators. The joint torques of the right leg are shown in Fig.13 for pitch hip, Fig. 15 for pitch knee, and 16 for pitch ankle. Also, kinematic model for finding the corresponding the left joint angles of hip, knee are shown in Figs. 12, and 14, respectively. Other simulation result isn't present in here. The trajectories are smooth enough for joint control. The gait trajectory planning and simulation interface using Matlab is shown in Fig. 17.
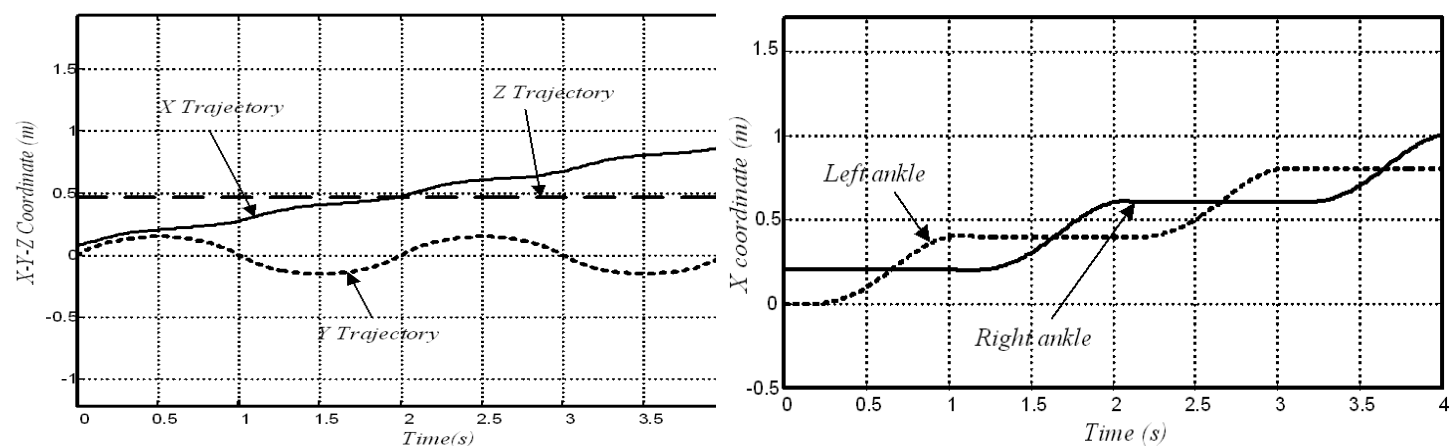

Fig.6. Hip trajectory

Fig. 7. Ankle trajectory in $X$ coordinates system 


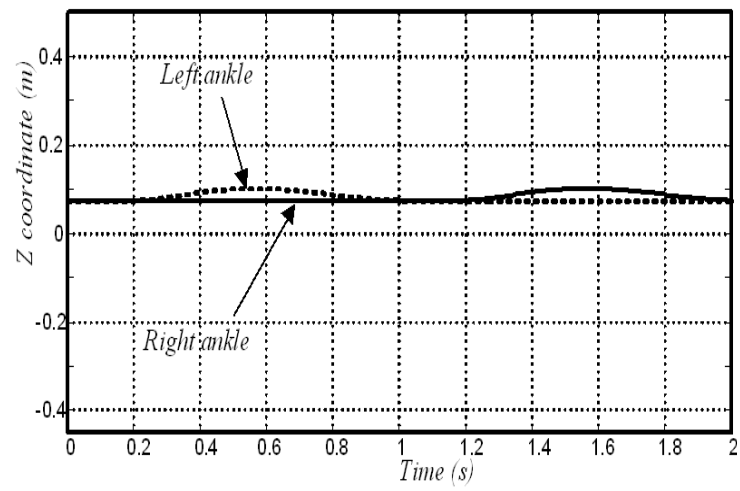

Fig. 8. Ankle trajectory in Z coordinates system

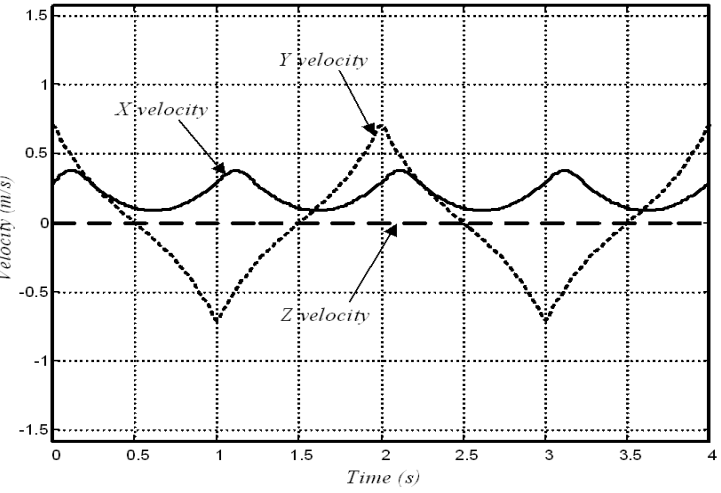

Fig. 9. Hip velocity
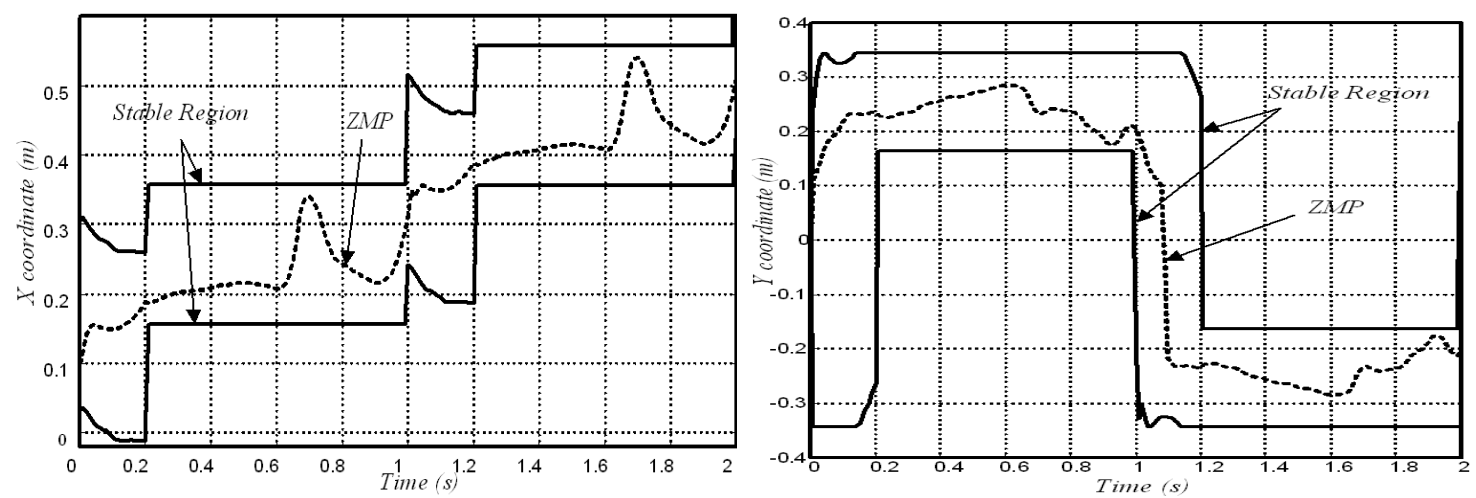

Fig. 10: ZMP trajectory a) in $\mathrm{X}$ coordinates system b) in $\mathrm{Y}$ coordinates system.

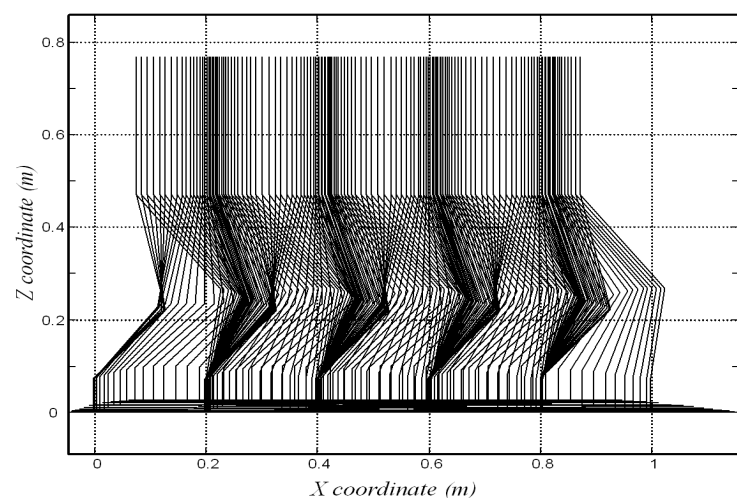

Fig.11. Full body trajectory

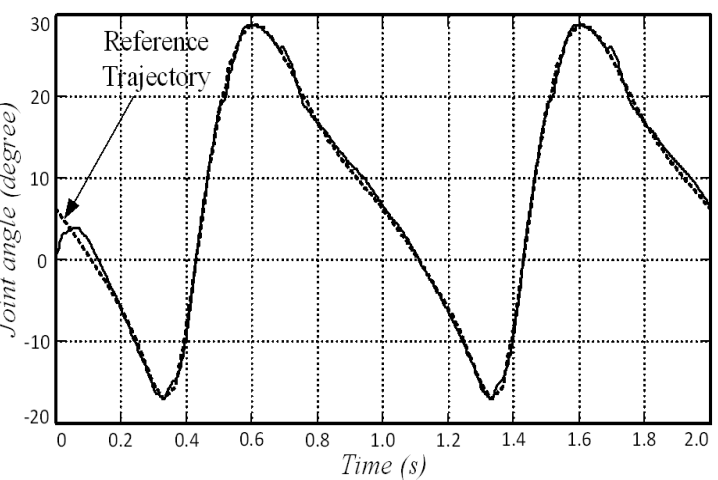

Fig.12. Pitch joint trajectory of right hip

Through the simulation, it is known that a high foot requires large peak torque and velocity of almost all the joints; that is to say, the higher the swing foot lifts, the larger the energy required to drive the joint is. In addition, the case of the low hip makes the robot walking faster than the case of high hip position. 


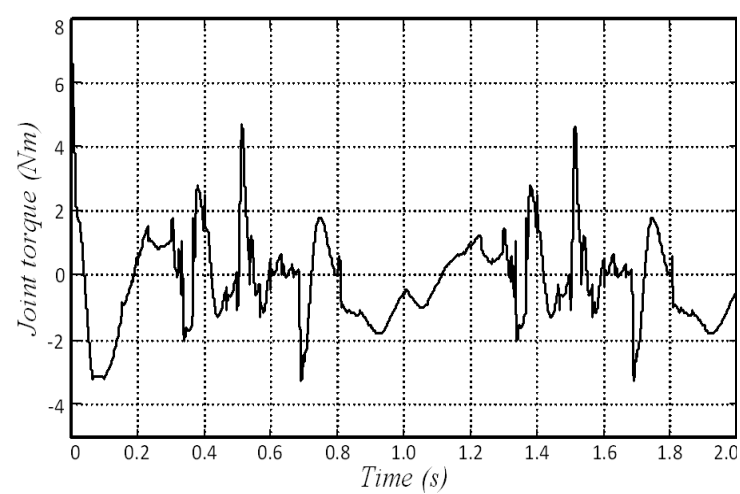

Fig.13. Pitch joint torque of right hip

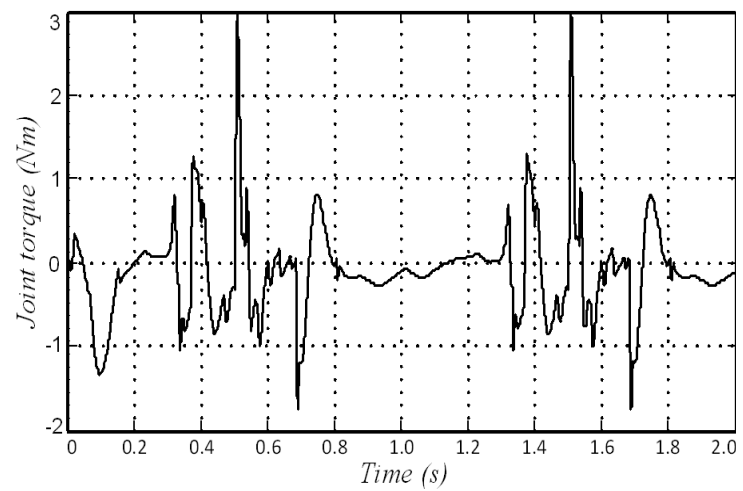

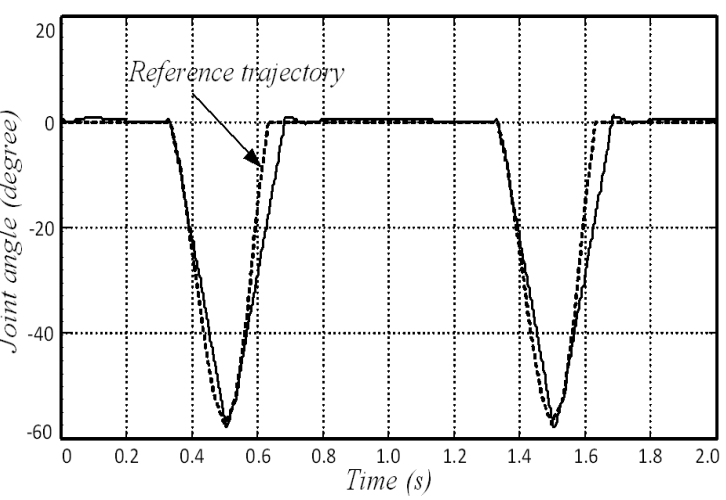

Fig.14. Right knee joint trajectory

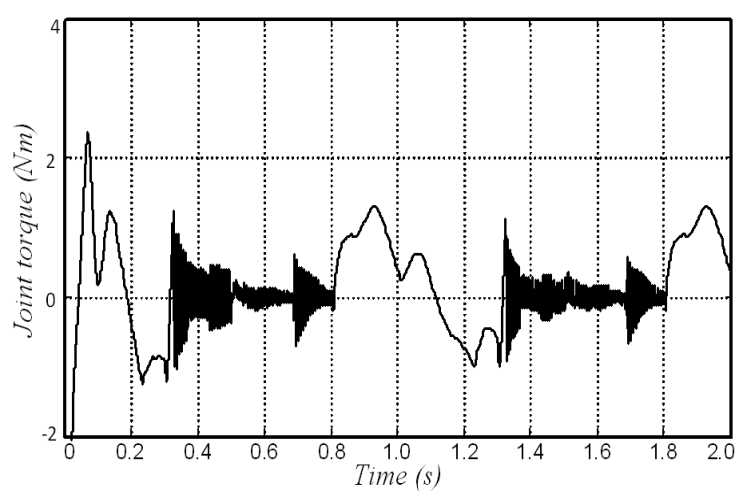

Fig.15. Right knee joint torque

Fig.16. Pitch joint torque of right ankle

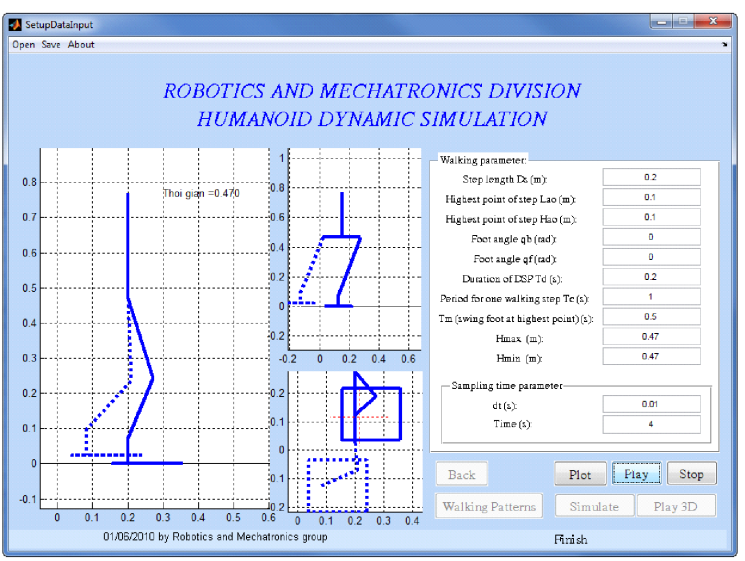

a)

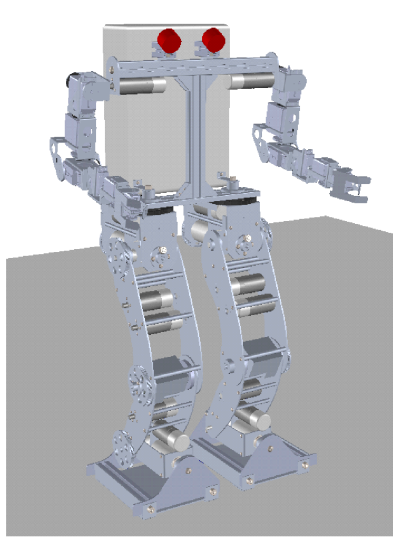

b)

Fig.17. Simulation interface of HUBOT-1

a) The gait trajectory planning and simulation; b) The dynamic simulation using MatlabSimulink. 


\section{CONCLUSION}

In this paper, the off-line method based on [10] for planning walking patterns has been applied to our biped robot HUBOT-1 in sagittal plane. Hip motion in y-dimensional is based on CoM trajectory from [12]. Human-like walking patterns is realized by analyzing human walk and then set the desired step length, foot angle, etc. according to this. The hip and ankle trajectories are planned according to the parameters of the robot and the ground conditions. Different foot motions are produced, and the motion which satisfies stable constraints is chosen for deriving the joint actuators. Simulation results have shown the walking planning successfully enables a stable bipedal walk without a fall.

\section{REFERENCES}

[1] Yu Ogura, Kazushi Shimomura, Hideki Kondo, Akitoshi Morishima, Tatsu Okubo, Shim-pei Momoki, Hun ok Lim, and Atsuo Takanishi, Human-like walking with knee stretched, heelcontact and toe-off motion by a humanoid robot, IEEE/RSJ International Conference on Intelligent Robots and Systems (Oct. 2006) 3976-3981.

[2] Klaus Loffler, Michael Gienger, and Friedrich Pfeiffer, Sensors and control concept of a biped robot, IEEE Transactions on Industrial Electronics (Oct. 2004) 972-980.

[3] M. Vukobratovic and D. Juricic, Contribution to the synthesis of biped gait, IEEE Trans. Bio-Med. Eng. vol. BME-16 (1) (1969) 1-6.

[4] F. Gubina, H. Hemami, and R. B. McGhee, On the dynamic stability of biped locomotion, IEEE Trans. Bio-Med. Eng. vol. BME-21 (2) (1974) 102-108.

[5] A. Takanishi, M. Ishida, Y. Yamazaki, and I. Kato, The realization of dynamic walking robot WL-10RD, Proc. Int. Conf. Advanced Robotics, 1985 (459-466).

[6] C. L. Shih, Y. Z. Li, S. Churng, T. T. Lee, andW. A. Cruver, Trajectory synthesis and physical admissibility for a biped robot during the singlesupport phase, Proc. IEEE Int. Conf. Robotics and Automation, 1990 (1646-1652).

[7] K. Hirai, M. Hirose, Y. Haikawa, and T. Takenaka, The development of honda humanoid robot, Proc. IEEE Int. Conf. Robotics and Automation, 1998 (1321-1326).

[8] A. Dasgupta and Y. Nakamura, Making feasible walking motion of humanoid robots from human motion capture data, Proc. IEEE Int. Conf. Robotics and Automation, 1999 (1044-1049).

[9] A. Takanishi, M. Ishida, Y. Yamazaki, and I. Kato, The realization of dynamic walking robot WL-10RD, Proc. Int. Conf. Advanced Robotics, 1985 (459-466).

[10] Qiang Huang, Kazuhito Yokoi, Shuuji Kajita, Kenji Kaneko, Hirohiko Arai, Noriho Koyachi and Kazuo Tanie, Planning walking patterns for a biped robot, IEEE Transactions on Robotics and Automation 17 (3) (June 2001).

[11] C. Shih, Gait synthesis for a biped robot, Robotica 15 (1997) 599-607. 
[12] K. Erbatur, O. Kurt, Natural ZMP trajectories for biped robot reference generation, IEEE Transactions on Industrial Electronics 56 (3) (March 2009).

[13] Vukobratovic, M. and Borovac, B., Zero-moment point - thirty five yearsof its life, Journal of Humanoid Robotics 1 (1) (2004) 157-173.

[14] TN Nguyen, HL Phan, TL Chung, Development of a humanoid biped walking robot Hubot-1, VCM National Conf. (2010).

[15] TN Nguyen, TL Chung, Walking planning for biped robot Hubot-1, VCM National Conf. (2010).

Received on October 25 - 2010 